\title{
Heavy precipitation events over a few Alpine sub-regions and the links with large-scale circulation, 1971-1995
}

\author{
Guy Plaut $^{1, *}$, Evi Schuepbach ${ }^{2}$, Marut Doctor ${ }^{2}$ \\ ${ }^{1}$ Institut Non Linéaire de Nice CNRS, Université de Nice-Sophia Antipolis, 1361 route des Lucioles, 06560 Valbonne, France \\ ${ }^{2}$ Climate and Background Ozone (CABO), Physical Geography, University of Berne, Switzerland
}

\begin{abstract}
Due to orographic effects, the European Alps are particularly sensitive to floods, in particular the ones following heavy precipitation events (HPEs). During recent years, there have been numerous damaging floods in the Alps, especially on the southern flank, but also intense snowfall, with attendant avalanches and loss of life on the northern flanks. Here, we objectively classify (in the space of their leading EOFs) the large-scale circulation (LSC) selected patterns giving rise to HPEs over alpine sub-regions. The introduction of a 'classifiability index' allows one to objectively choose the best number of clusters. Strikingly robust cluster centroids are found in most cases. Some of the clusters have a high 'discriminating power': whenever a daily LSC looks similar enough to the centroid of one of these clusters, the probability of an HPE is highly enhanced. This suggests building downscaling algorithms and semi-empirical HPE forecast schemes. The latter would be based on a comparison between the dynamically forecasted LSCs and the cluster centroids and would benefit both from the present skill of LSC forecasts (LSC forecasts are often better than direct precipitation forecasts, especially in mountainous terrains) and from the existence of cluster centroids with high discriminating power. The downscaling algorithms could also provide accurate tools for the examination of the possible small-scale consequences of global change, as simulated by GCMs at a larger scale. The question of the robustness of the cluster centroids is hence an important one and is further investigated through sensitivity experiments. Using half-period precipitation data, the same patterns are found. The precise number of EOFs kept to perform the classification is also irrelevant. Classification of HPE LSCs into clusters is also compared to alternative approaches.
\end{abstract}

KEY WORDS: Classification · Large-scale circulation $\cdot$ Heavy precipitation event $\cdot$ Downscaling

\section{INTRODUCTION}

Among all natural hazards, floods cause the greatest material damage in the world. Due to orographic effects, the European Alps are particularly sensitive to floods, especially the ones following heavy precipitation events (HPE). In recent years, and especially during last fall and early winter, there have been numerous HPEs over the southern flanks of the Alpine region, which resulted in destructive floods with

*E-mail: plaut@inln.cnrs.fr extended damage and loss of life. Recently, Massacand et al. (1998) reported on a study on 4 extreme events: Vaison-la-Romaine (September 1992), Italian Piedmont (and south-eastern France) (November 1994), Brig (September 1993), and South Ticino (September 1995). The objective was to explore whether these events possessed an upper-level identifiable precursor signal, with a focus on the distribution of potential vorticity (PV). It was found that the 4 events were accompanied by strikingly similar PV patterns characterized by meridionally elongated deep intrusions of stratospheric air. Corresponding to this PV anomaly, the upper-level flow was characterized by a large ampli- 
tude wave in the $500 \mathrm{hPa}$ height field (Feretti et al. 2000), with an eastward moving trough typically extending northwards from Spain to the British Isles at the onset time of heavy precipitation. Intriguingly, these studies do not comment on the presence of an upper-level ridge to the west of the trough (and PVanomaly streamer), sometimes extending northwards up to south Greenland. This ridge ensures cold midtropospheric air advection, also a factor favouring intense precipitation through extended cumulus convection (Emmanuel 1991). Feretti et al. (2000) noted the presence of an almost stationary downstream ridge over eastern Europe, which contributes to blocking the perturbation over western Mediterranean areas and to contracting the warm tongue in the $850 \mathrm{hPa}$ temperature field. Of particular importance is the eastward progression of the moist air stream to locations where one might expect strong orographic enhancement of precipitation as the southerly flow impinges on the southern flanks of the Alps. Doswell et al. (1998) and the simulations by Buzzi et al. (1998) and Romero et al. (1998) provide evidence for the importance of both the large-scale dynamics and the embedded, orographically enhanced cumulus convection.

All the studies in the published literature are limited to a few particularly intense HPEs. This paper aims to provide a comprehensive analysis and systematizes, whenever possible, case study observations. For this purpose, we consider the set of large-scale circulation patterns (LSC) which give rise to HPEs over defined Alpine sub-regions such as Ticino and the French Alpes Maritimes. That is to say, we select the LSCs (namely the $Z 700$ height over the North Atlantic and western Europe) of the HPE days and apply the dynamical cluster algorithm, which reduces them into a few classes or clusters. For instance, if all events were due to the presence of an elongated PV streamer such as demonstrated by Massacand et al. (1998), we would expect the most stable classification to be one single cluster exhibiting this corresponding large amplitude wave pattern. However, there may be different kinds of large-scale patterns giving rise to HPEs over a defined Alpine sub-region, and in such cases, one can expect the best classification to consist of several clusters.

The investigation of the possible links between LSCs and local weather was one of the main tasks in the EC contract ACCORD (ENV4-CT97-0530). Until the late 1970s, the most famous classification schemes such as the 'Grosswetterlagen' approach developed in Germany (Hess \& Brezowsky 1952, 1969) or the Lamb weather types (Lamb 1972), widely used by British synopticians, remained mostly subjective. Later, the generic name of 'weather regimes' was attached to the (a priori unknown, unlike the previous schemes) clus- ter centroids that one obtains through fully objective classification procedures. The algorithms leading to classification of daily LSCs into weather regimes may be approximately gathered into 3 categories (Plaut \& Simonnet 2001, this issue), depending on which characteristic of the most recurrent large-scale patterns is emphasized (recurrence or quasi-stationarity for instance). However, in all cases, classifications into weather regimes take all available circulation patterns into account. Here, in contrast, we only classify LSCs which give rise to heavy precipitation over a given Alpine sub-region. Our ultimate aim would be to obtain patterns with a high 'discriminating power'. Hence, we would like to find that whenever the LSC pattern is similar enough to one cluster centroid, an HPE is actually very likely. Such considerations have, of course, to be quantified. Moreover, we cannot expect that one single large-scale field holds enough information to obtain a very high discriminating power. However, in view of the importance of the large-scale dynamics in cases where orographically enhanced convection (Ferretti et al. 2000) plays a major role, one might expect relatively high discriminating power in the most favourable cases.

The generic name of 'downscaling' (Hay et al. 1991, Bardossy \& Plate 1992, Wilby 1998) is usually attached to algorithms which allow one to infer (mostly in a statistical sense) local weather starting from (more or less) LSC patterns. Downscaling algorithms take advantage of the invoked (statistical) link between LSCs and local weather. In particular, the weather generation games with the so-called stochastic weather models (Katz \& Parlange 1996, Wilks \& Wilby 1999) are typical applications of downscaling. They are commonly used to simulate the succession of local weather types in a realistic way, with application, for instance, to hydrological studies. Downscaling also makes it possible to simulate local weather using general circulation model (GCM) output by employing the statistical knowledge of the stochastic links between large-scale patterns and local weather (Wilby et al. 1998). These links are in principle deducible from observations. Provided they remain stationary in the near future, downscaling may be used to explore the mesoscale manifestations of global change starting from GCM simulations.

Downscaling may, however, have more immediate applications in specific cases. Any synoptician may ascertain that the presently available operational models provide more accurate forecasts of air mass circulation than of precipitation, especially over mountainous areas. But it could actually occur, especially over such areas, that the links between LSC and the local weather are strong enough to permit useful downscaling-type precipitation forecasts. This would be the case if, for instance, meridionally elongated PV 
streamers, such as those demonstrated by Massacand et al. (1998), were always accompanied by heavy precipitation over the Mediterranean flanks of the western Alps. The ultimate goal of our study of HPEs through the classification of the corresponding LSCs into clusters would actually lie in the elaboration of downscaling-type HPE forecast algorithms. The possibility that such algorithms may be constructed requires that the isolated LSC clusters hold a rather high discriminating power, as discussed above.

The raingauge data we use, as well as the LSC data, are described in Section 2, where we also discuss the definition of HPEs and briefly describe the dynamical cluster algorithm. Section 3 is devoted to a detailed study of HPEs over 2 contrasted regions in the French Alps: the Alpes Maritimes and Savoy. The case of an intermediate region, the Queyras, is also briefly discussed, and the robustness of the classes is checked from various points of views. In Section 4, we concentrate on a few other Alpine and Mediterranean subregions and compare the cluster centroids one obtains for different (southern) Alpine or Mediterranean subregions. Recent trends are also discussed. Before the conclusions in Section 6, alternative approaches to HPE studies and their semi-empirical forecasts are discussed in Section 5, including the more classical composite techniques, or the use of the weather regimes (Plaut \& Simonnet 2001).

\section{DATA PROCESSING AND METHODOLOGY}

\subsection{Data}

To investigate heavy regional precipitations over the Alps, we use $25 \mathrm{yr}$ of dense raingauge data kindly provided by the Swiss Federal Institute of Technology, Zürich: the 'Alpine Precipitation Climatology' (Frei \& Schär 1998) covers the European Alps area and the adjacent foreland. It consists of a compilation of 6678 station records projected on a $25 \mathrm{~km}$ grid for each day of the $25 \mathrm{yr}$ period from 1971 to 1995 . Each grid point corresponds to an average of 6 stations. These data offer very attractive possibilities for investigations of intense precipitation on a small scale.

The large-scale fields we use for automatic classification of LSCs corresponding to HPEs consist of a set of re-analysed $700 \mathrm{hPa}$ height fields. They were obtained from the NCEP/NCAR reanalysis project and extend from 1958 to 1998 (Kalnay et al. 1996). They were spatially extracted at the Climatic Research Unit, Norwich, UK. As in Plaut \& Simonnet 2001, we use a $100^{\circ}$ longitude, $40^{\circ}$ latitude wide window centered at $50^{\circ} \mathrm{N}, 10^{\circ} \mathrm{W}$; it therefore covers most of the North Atlantic and Europe. Before proceeding to the classifi- cation, we first remove the seasonal cycle and a seasonal linear trend at each grid point; then a PCA is performed in order to reduce the number of spatial dimensions without loss of useful information.

\subsection{Definition of HPEs}

HPEs over the Alps most often affect rather small sub-regions. For instance, intense precipitation over Savoy is usually accompanied by dry sunny weather over the southern Alps. Even Languedoc-Roussillon and the Côte d'Azur almost never simultaneously receive heavy rains. We therefore define small Alpine subregions typically corresponding to $10-15$ grid points of the 'Alpine Precipitation Climatology' (Fig. 1). HPEs over one of these sub-regions then have to be defined by some objective criterion. The fact that each grid point contains information from 5 or 6 stations excludes days from the HPE set where only 1 or 2 stations registered heavy precipitation. We considered this as an advantage as we are ultimately interested in identifying potentially damaging events, since, most often, very localized heavy precipitation has limited hydrological consequences. Now, 2 possibilities remain: the statistical distribution may itself define the threshold of heavy precipitation (e.g. one may use the 95th percentile value at each grid point), or we may choose the same specific threshold everywhere. Both choices have advantages and disadvantages. Here, we prefer to choose a specific threshold: we say that an HPE occurs over a given region on any day when at least one of the grid points in this region receives more

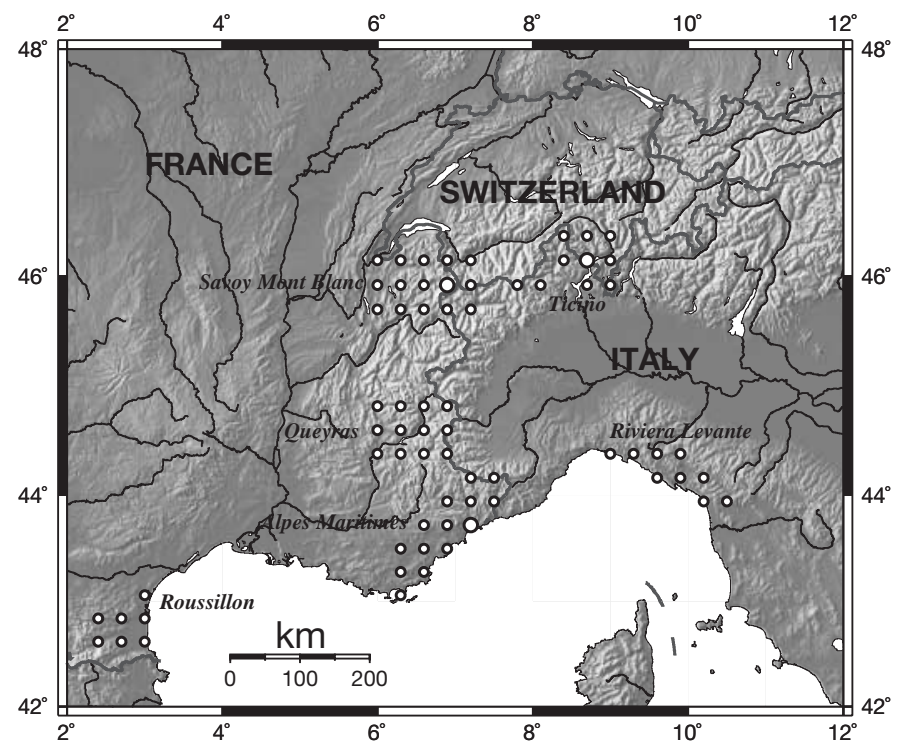

Fig. 1. The Alpine Precipitation Climatology gridpoints defining each of our sub-alpine domains 
than a fixed precipitation threshold. With this definition, there will of course be more HPEs for wet regions such as Ticino than for arid massifs such as Queyras, this difference reflecting an hydrological fact. We verified that the exact value of the threshold was irrelevant. With the rather commonly used threshold of $40 \mathrm{~mm}$, we are left with about 12 HPEs per year in the Alpes Maritimes, which roughly corresponds to events having generated some damage. Raising the threshold value to e.g. $50 \mathrm{~mm}$, although leaving one with less HPEs, leads to fully indistinguishable LSC cluster centroids.

\subsection{Classification of LSCs ( $Z 700$ heights patterns)}

Once the HPEs are identified for a given Alpine subregion, we select the corresponding LSCs and classify them into clusters using the dynamical cluster algorithm (Michelangeli et al. 1995), as implemented in our package ANAXV. Classification is performed within the 10-dimensional space of the 10 leading principle components (PCs) of the $Z 700$ geopotential heights; the robustness of the cluster centroids against changes in this dimensional parameter is discussed in Section 5.1.

An important question when performing clustering is the number of clusters required (Michelangeli et al. 1995). We try to answer this question in the following way: a significance test checking the robustness of the classifications for each value of $k$, the number of clusters, is defined. The optimal value of $k$ should be determined by the data themselves, following objective criteria. With this optimal value of $k$, different classifications will not lead to non-robust patterns for the cluster centroids (a pattern is robust if it does not depend on the details of the classification procedure, nor especially on the random seeds selected to initiate the procedure; see below). In order to make this requirement more quantitative, we introduce a 'classifiability index' $C^{*}$. The present test is derived from the random noise test of Michelangeli et al. (1995) described in Plaut \& Simonnet 2001. Here we briefly summarize the procedure. Starting from random seeds taken among the patterns to be classified, the dynamical cluster algorithm finds partitions of these patterns into clusters. Let $P$ and $Q$ be 2 such partitions into $k$ clusters starting from different random seeds. If $k$ has its optimal value, and if the classifiability is good, $P$ and $Q$ will be very similar whatever the initial seeds. This should result in a high value for the classifiability index. In practice, we first define an anomaly correlation coefficient between 2 partitions: $C(P, Q)$, which is equal to 1 if the 2 sets of cluster centroids are identical and -1 in the worst case; $C(P, Q)$ measures the correlation of the worst matched pair when matching $P$ and $Q$ into corresponding pat- terns. $N$ classifications of the selected LSCs are achieved, and $C^{*}$ is ultimately defined as the average of the $N(N-1)$ values one obtains for $C(P, Q)$. In order to lay down significance limits for $C^{*}, 100$ sets of the same number of LSCs as the HPE LSCs to be classified are randomly selected from the full LSC data set. They give 100 values of $C^{*}$ that are ranked to find the $90 \%$ confidence limits. Then we compare the index $C^{*}$ of the true HPE LSCs with these bounds: values above the upper bound indicate a classifiability significantly higher than for the randomly selected LSCs. Most often, this test unambiguously settles the number of clusters. Even with values of $C^{*}$ around the $90 \%$ confidence limit, the similarity of the cluster centroids one obtains for different classifications is striking.

\subsection{The discriminating power of a cluster}

In order to estimate the usefulness of a classification we introduce the concept of 'discriminating power'. We ask ourselves if any practical conclusion may be firmly stated when the LSC (analysed, or even forecasted) resembles one of the cluster centroids. If this is the case, that is, if patterns quite similar to a given cluster centroid correspond to HPEs with a highly enhanced probability, and also lead to high precipitation composites over the given sub-domain, the involved cluster has a high discriminating power. To make this requirement more quantitative, HPE conditional probability histograms and precipitation composites are displayed against the anomaly pattern correlation between any given day $Z 700$ height field and that of the cluster centroids.

\section{HPES OVER THE ALPES MARITIMES, SAVOY-MONT BLANC, AND QUEYRAS}

We first proceed to the classification of LSCs leading to HPEs over 3 contrasted sub-regions of the French Alps: the Alpes Maritimes lie on their Mediterranean flank, whereas Savoy-Mont Blanc is situated on the opposite side; the so-called Queyras is an intermediate massif which records a much smaller amount of heavy precipitation. The grid points covering these regions can be seen on Fig. 1, together with the orography of the Alps and the adjacent foreland. Note the steep orography of the Alpes Maritimes.

\subsection{LSC clusters for HPEs over the Alpes Maritimes}

Whereas this sub-region starts at sea level along the French Riviera, elevations may reach $3000 \mathrm{~m}$ at the 
a)
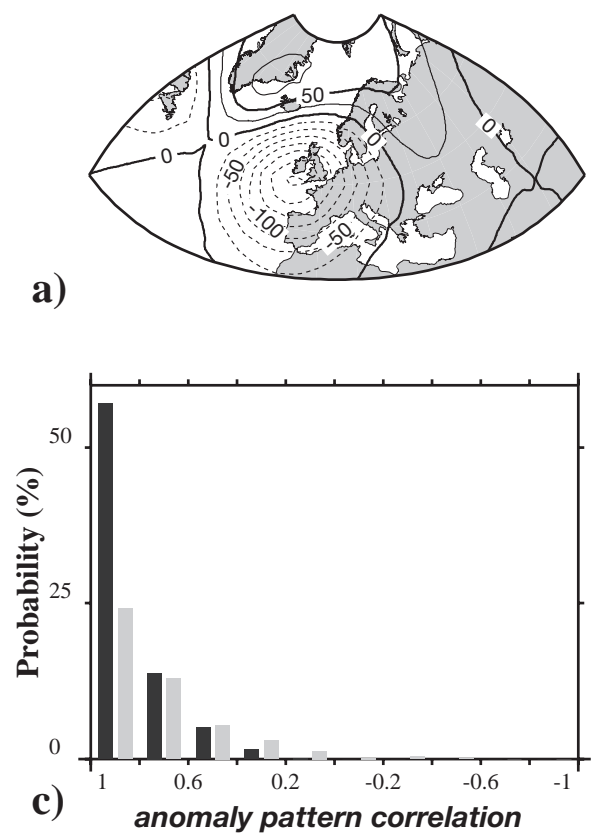

b)
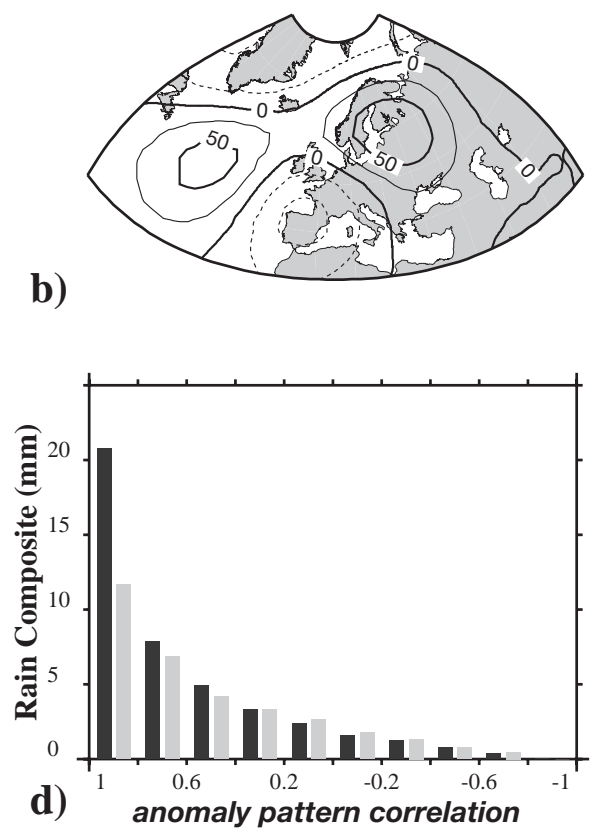

Fig. 2. (a,b) Geopotential anomaly map of cluster centroids for HPEs over the Alpes Maritimes; (c) conditional probability of any day being a class 1 (black bars) or a class 2 (grey bars) HPE, as a function of the anomaly pattern correlation (apcc) between this day's LSC and the corresponding cluster centroid; (d) the same, but for all of the days of the 25 yr precipitation composites at the Alpes Maritimes domain grid point marked with a larger circle in Fig. 1

grid points furthest north (over the so-called 'Massif du Mercantour'). As a consequence, southerly and southeasterly flows may cause severe hazards in this region. With our definition of HPEs (Section 2.2), one obtains about 12 HPEs per year over the Alpes Maritimes during the period 1971-1995. Once these episodes have been identified, we proceed to the classification of the corresponding LSCs in the space spanned by their 10 leading PCs. Our random test (Section 2.3) selects $k=$ 2 clusters with a classifiability index $c^{*}$ well above the $90 \%$ significance limit: independent classifications starting from different random seeds always lead to fully identical clusters.

The cluster 1 centroid anomaly map (Fig. 2a) displays a positive (anticyclonic) anomaly over southern Greenland, together with a deep negative anomaly south of Ireland near Sole. These anomalies generate a large amplitude wave at the mid-tropospheric level, as pointed out in Ferretti et al. (2000) for the Piedmont flood of November 1994; they advect anomalous southwesterlies over the southern Alps, but also anomalous north-westerlies over the central north Atlantic. We term this cluster GASC (Greenland anticyclone-Sole cyclone). It has a remarkable discriminating power, as can be seen in the histogram of Fig. 2c (black bars). More than $50 \%$ of LSCs having, within their 10-dimensional leading PC space, an anomaly pattern correlation coefficient, apcC $>0.8$, with the GASC pattern of
Fig. 2, lead to HPEs. It is worth adding that if one categorizes the intensity of the anomaly field into 3 equally populated terciles (above, median, and below), the conditional probability of HPE for LSCs having an apcC $>0.8$ with the GASC centroid even rises close to $70 \%$ for the above category. Given these high percentages, downscaling-type approaches to heavy precipitation semi-empirical forecasts could appear very attractive. They could, for instance, be based on the dynamically forecasted LSCs, and hence use could be made of the good quality of present-day operational models. Indeed, in fall 2000 we verified the high quality of the middle-range operational forecasts of GASC-like patterns for lead-times of up to 5 or 6 d before damaging events.

The discriminating power of a cluster can also be investigated through the computation of precipitation composites. The composites of Fig. 2d correspond to the grid point closest to the city of Nice (larger circle in the Alpes Maritimes on Fig. 1). They lead to conclusions similar to those which may be drawn from Fig. 2c and confirm the power of the GASC cluster. Another important characteristic of a cluster of LSCs leading to HPEs is its seasonal behaviour. Fig. 3 shows (upper histogram, black bars) that 'GASC' events are predominantly autumn or cold season events. Such HPEs may bring about heavy snowfalls over the southern Alps, as was the case on 25 December 2000, or during January 


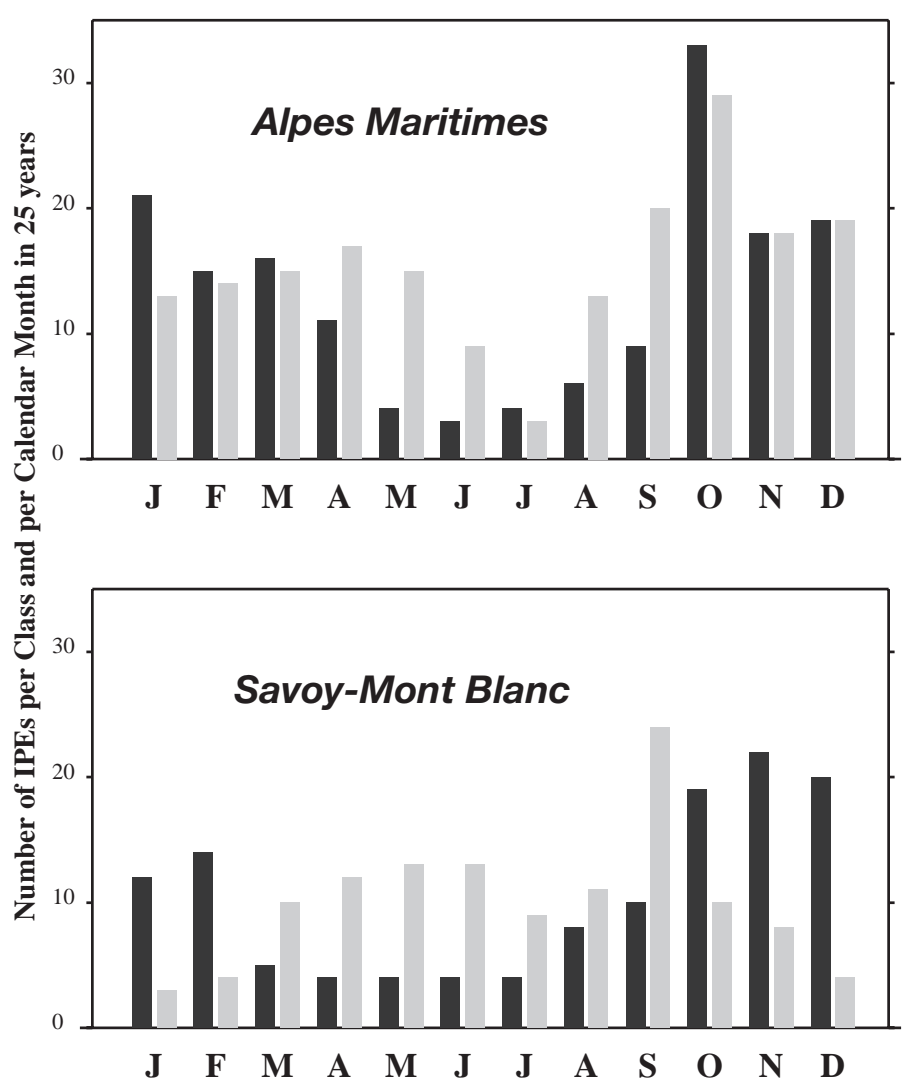

Fig. 3. Number of occurrences per calendar month of class 1 (black bars) and class 2 (grey bars) HPEs for both the Savoy-Mont Blanc and the Alpes Maritimes

1994, when 4 HPEs of the GASC type occurred, giving rise to avalanches and extensive damage to power lines in mountainous areas of the Alpes Maritimes.

A comment about actual synoptic maps leading to HPEs must be added here: since the classification has been achieved within the 10-dimensional leading PC space, the full synoptic maps of HPEs may involve additional smaller-scale patterns which are absent from the cluster centroids. In particular, the flow over the southern Alps will appear more southerly on these maps. Taking also into account these smaller regional scales could probably allow one to improve the discriminating power, although this is beyond the scope of the present paper. Moreover, it would not be possible to achieve significant classification of a few hundred patterns within a too large dimensional space; taking smaller scales into account would, of course, increase the dimension of the EOF space in which the classification is performed.

Cluster 2 could be termed QP (quadrupole); with this cluster, the HPE conditional probabilities do not take that high values, as shown by the grey bars on lower histograms of Fig. 2: the discriminating power of QP is obviously much lower, and its pattern does not appear very attractive from the point of view of semi-empirical HPE forecasting. It may be enlightening to mention that if one tries classification into 3 clusters, one still obtains quite robust patterns (see Fig. 7 and Section 4.1). For larger numbers of clusters $(k>3)$, whereas the GASC pattern remains quite robust, cluster 2 (QP) is most often broken into smaller (less populated) non-robust clusters: their centroids depend on the random seeds used to start the classification procedure (our methodological point of view is explained in Section 2.3). The cluster 2 centroid could perhaps be looked at as a weighted average between a few fuzzy (illdefined) sub-clusters, whereas the cluster 1 (GASC) centroid appears as a real LSC pattern leading to HPEs with a high conditional probability.

\subsection{LSC clusters for HPEs over Savoy-Mont Blanc}

Like that for the Alpes Maritimes, the random significance test for the Savoy-Mont Blanc selects $k=2$ for the best number of clusters. Their centroids are displayed in Fig. 4a,b. The most outstanding feature of cluster 1 is a strong negative height anomaly $(-150 \mathrm{~m})$ over the North Sea, whereas cluster 2 may be characterized by its weak negative anomaly over western Europe, with a light anomalous southerly flow over the Alps. Daily anomalies almost never look very similar to the cluster 2 centroid since there are only 10 days (during 25 yr) with an apcc $>0.8$ with the cluster 2 centroid, none of them being an HPE. Looking at grey bars in Fig. 4, one realizes that this cluster has a very low discriminating power: it may be viewed as an aggregate of scattered LSC patterns leading to heavy precipitation mainly from March to early fall (Fig. 3), with a pronounced peak in September.

On the contrary, anomaly patterns similar to that of the cluster 1 centroid do occur in the actual anomaly maps. They mostly correspond to rather heavy precipitation days: more than a quarter of those days for which the LSC anomaly pattern correlation with cluster 1 centroid apcc $>0.8$ actually correspond to HPEs. The corresponding histogram of rain composites (Fig. 4, black bars) display a pronounced peak for the highest apcc values. Most of the (late) fall and winter heavy precipitation corresponds to class 1 HPEs (Fig. 3, lower histogram, black bars), in such a way that this cluster is responsible for the great bulk of heavy snowfalls. Patterns such as cluster 1 prevailed during the damaging 
a)
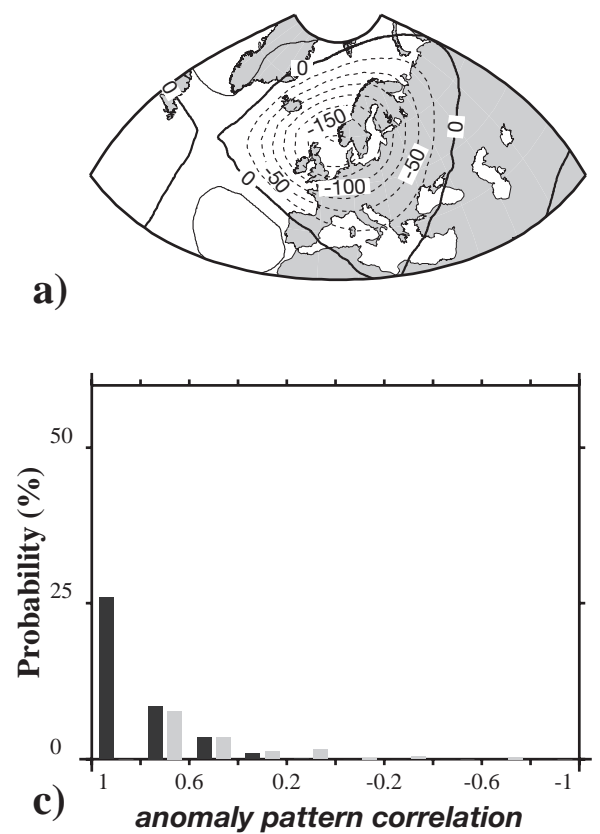

b)
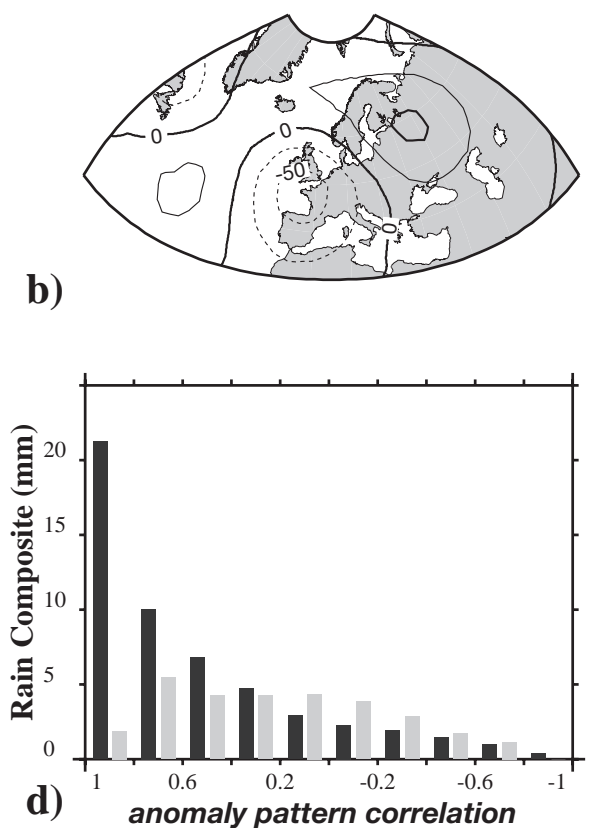

Fig. 4. (a,b) Geopotential anomaly map of cluster centroids for HPEs over the Savoy-Mont Blanc; (c) conditional probability of any day being a class 1 (black bars) or a class 2 (grey bars) HPE, as a function of the anomaly pattern correlation (apcc) between this day's LSC and the corresponding cluster centroid; (d) the same, but for all of the days of the 25 yr precipitation composites at the Savoy-Mont Blanc domain grid point marked with a larger circle in Fig. 1

avalanches of February 1999, which led to extensive loss of life in the French, Swiss, and Austrian Alps.

\subsection{HPE trends}

If one keeps in mind that the recent northern hemisphere warming mainly occurred after 1980 (Jones 1994), looking at intense precipitation trends during the $25 \mathrm{yr}$ between 1971 and 1995 may be sensible. We hence tentatively turn to a discussion of possible trends appearing either in HPE frequency, or in total precipitation amounts from HPEs. In order to obtain more significant conclusions, we consider average precipitation amounts for the whole set of grid points of an Alpine sub-region. In this way possible trends will be more confidently established, since local extreme events will be mostly dissolved within yearly averages involving 10 to 15 grid points and almost as many HPEs. In addition the precipitation amount at each grid point stands for a weighted average already involving several raingauge stations.

Opposite linear trends appear over the period 1971-1995 (Fig. 5). Annual precipitation due to HPEs are roughly 2.5 times larger in the Alpes Maritimes than over the Savoy-Mont Blanc region in the beginning of the $25 \mathrm{yr}$ period, while they exhibit similar values over both sub-regions at the end of the period. In the Savoy-Mont Blanc (which is located on the north- ern flank of the Alps), both the frequency of HPEs, and the corresponding precipitation amounts increased over this recent 25 yr period. Similar observations have been made in the north-western European countries, whereas the reverse tendency is found over the Alpes Maritimes, and the ecological risks may become worrysome if such trend $(-150 \mathrm{~mm}$ between the beginning and the end of the period) were to continue. Furthermore, if the precipitation records of the more recent years were available for analysis, the negative tendencies would appear more pronounced, at least until the wet (but atypic?) record-breaking winter of 2000-2001. But let us limit ourselves to the 1971-1995 period and mention that the total precipitation (i.e. the annual amounts from all forms of precipitation) displays even more pronounced opposite trends for Savoy-Mont Blanc $(+320 \mathrm{~mm}$ in $25 \mathrm{yr})$ and the Alpes Maritimes (-195 $\mathrm{mm}$ during the same period). Thus, the same conclusions stand for both total precipitation and HPEs.

\subsection{Comparison with Queyras}

In order to complete this comparative study, we performed a similar classification of LSCs corresponding to HPEs over an intermediate Alpine sub-region, the socalled Queyras and surrounding massifs (Fig. 1). Since the Queyras is sheltered by other massifs both from 

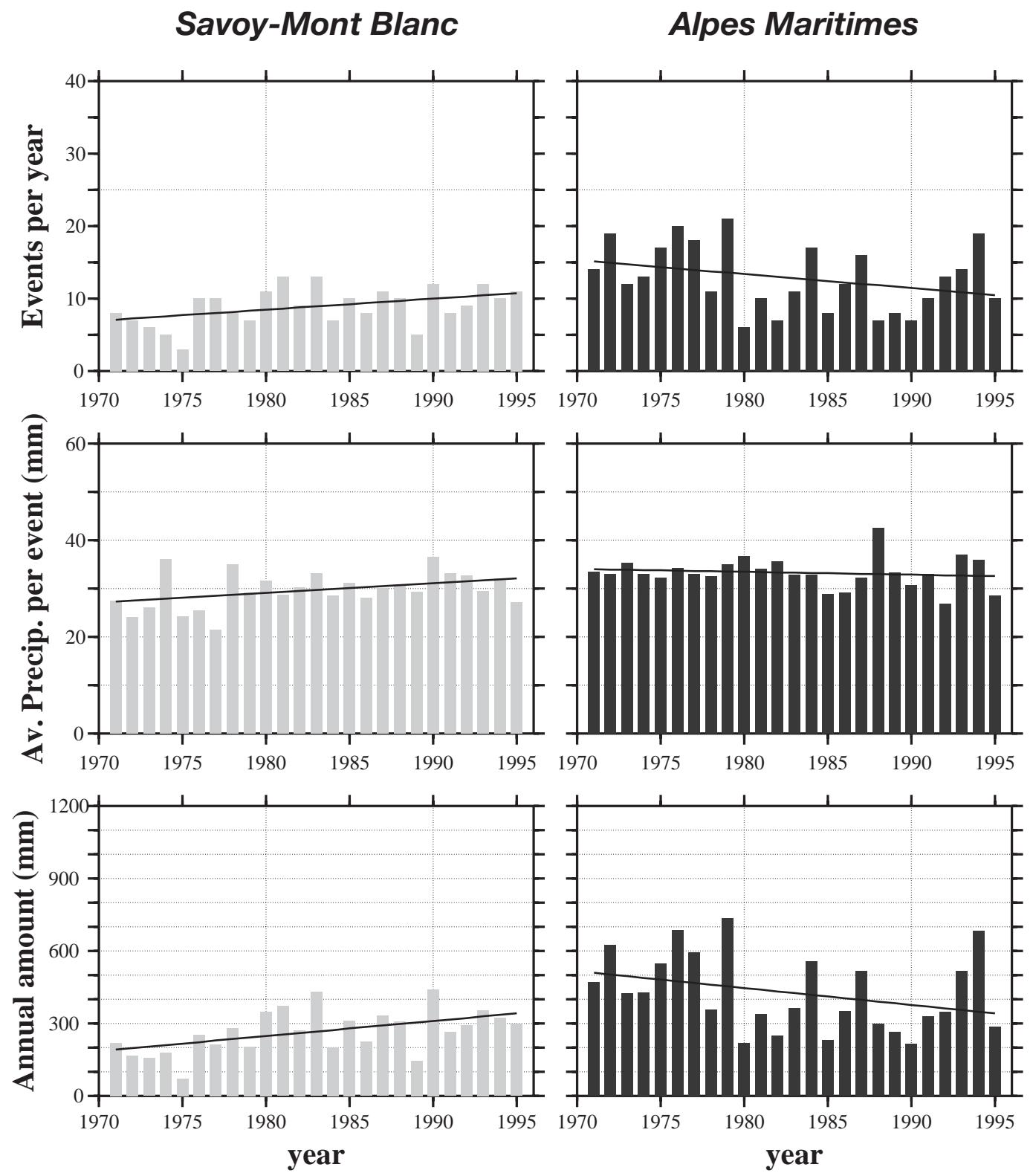

\section{Alpes Maritimes}
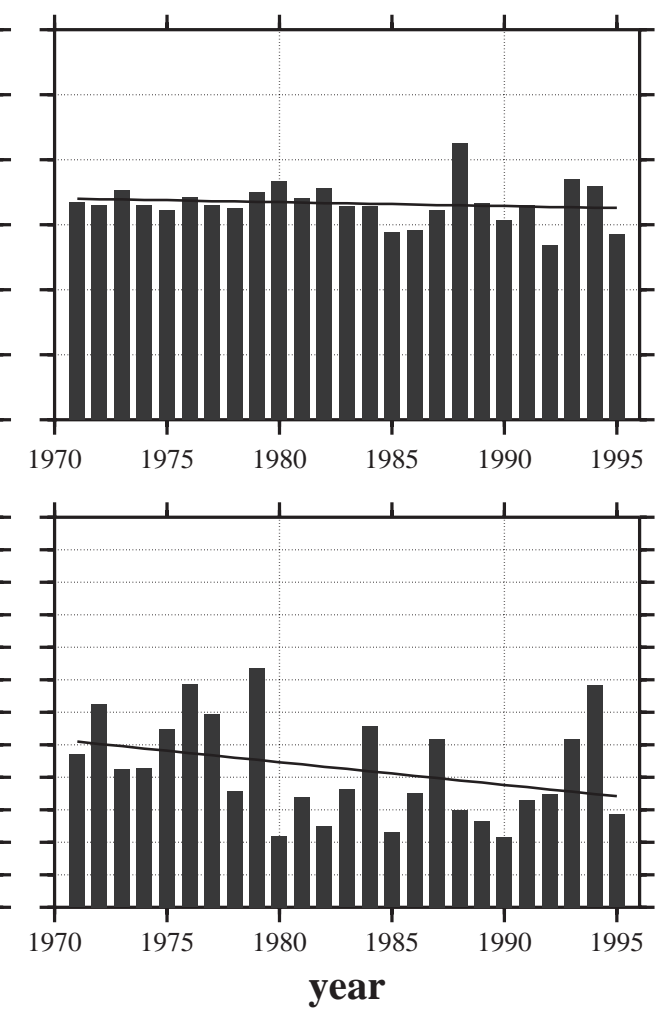

Fig. 5. Number of HPEs per year (top); average precipitation per HPE over the domain (middle), and total annual amount from HPEs, also averaged over the domain gridpoints (bottom), together with the trends. Data from the ETH Zürich Alpine Precipitation Climatology

northerly and southerly flows, not many HPEs can be expected, at least if the same threshold for the definition of HPEs is selected (Section 2.2). The classifications of HPE LSCs for the Queyras shows that $k=3$ is the best choice for the number of clusters. Fig. 6 displays some features of clusters 1 and 2, the most interesting ones for our purpose, indicating that annual heavy precipitation amounts are indeed much lower than for the previously investigated Alpine sub-regions. However, we feel it enlightening to observe that the Queyras cluster 1 centroid looks similar to the Savoy-Mont Blanc cluster 1 centroid, whereas the Queyras cluster 2 is almost identical to the Alpes Maritimes cluster 1 (GASC). In this way, one should not be amazed to observe the opposite linear trends exhibited by these 2 clusters' contributions to intense precipitation.

\section{LSC CLUSTERS FOR HPES OVER OTHER ALPINE SUB-REGIONS}

The analysis carried out for the Alpes Maritimes and Savoy-Mont Blanc was also done for 3 other Alpine sub-regions: Ticino, Roussillon in France, and the 

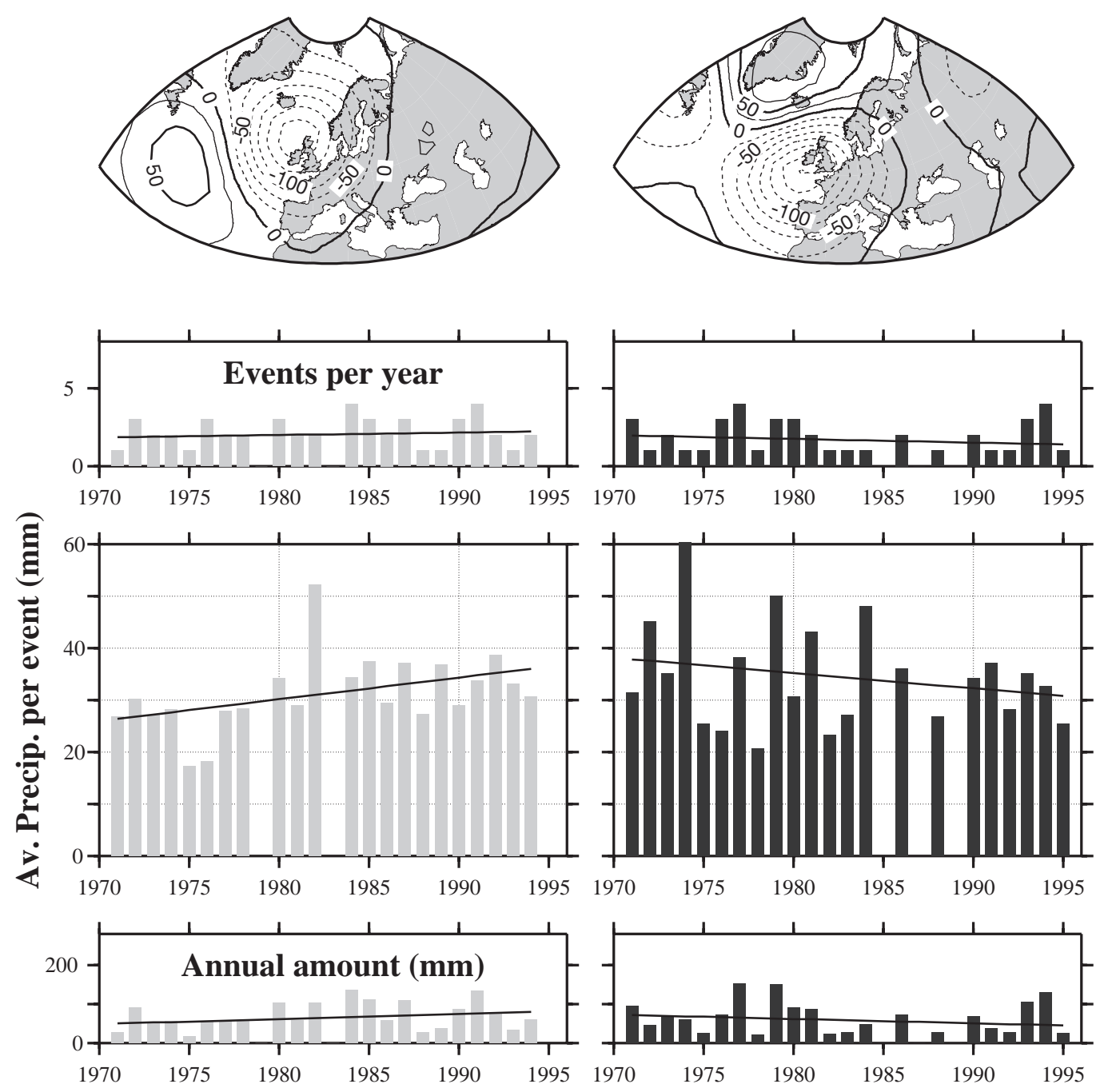

Fig. 6. Anomaly maps and yearly histograms for 2 classes of HPEs over the Queyras

Italian Riviera Levante, the latter two being Mediterranean border regions.

\subsection{Ticino}

The Lago Maggiore region, which covers northwestern Italy and the southern Swiss Alps, is one of the areas, in addition to the Julian and Carnic Alps, with the highest precipitation (Frei \& Schär 1998). Yearly maximum values reach $2500 \mathrm{~mm}$ in this region (Widmann 1996), while for the Alpes Maritimes they do not exceed $1500 \mathrm{~mm}$. The precipitation minimum is in winter for the Lago Maggiore region, but in summer in the Alpes Maritimes.

We first selected a rather wide domain around the Lago Maggiore, with 17 gridpoints lying on both sides of the mountain ridge between the Rhone valley and
Ticino. But the classifiability test (Section 2.3) indicated that the $90 \%$ significance level could not be reached. Then we reduced the number of grid points to 10 in order to encompass the region south of the Alps only (Fig. 1), and the classification into 3 clusters was found to become significant. The left-hand column maps of Fig. 7 display the 3 LSC cluster centroids for HPEs over this region. For comparison, the corresponding patterns for the Alpes Maritimes are displayed in the right-hand column: although 2 classes were preferred for the latter sub-region (Section 3.1), the cluster centroids for classification into 3 classes are also quite robust, despite a lower significance level. The Ticino cluster 1 centroid is similar to the Alpes Maritimes 'GASC' cluster displayed opposite (the same pattern as with 2 classes). Both cluster 2 and cluster 3 can unambiguously be associated with rather similar patterns corresponding to HPEs over the Alpes 

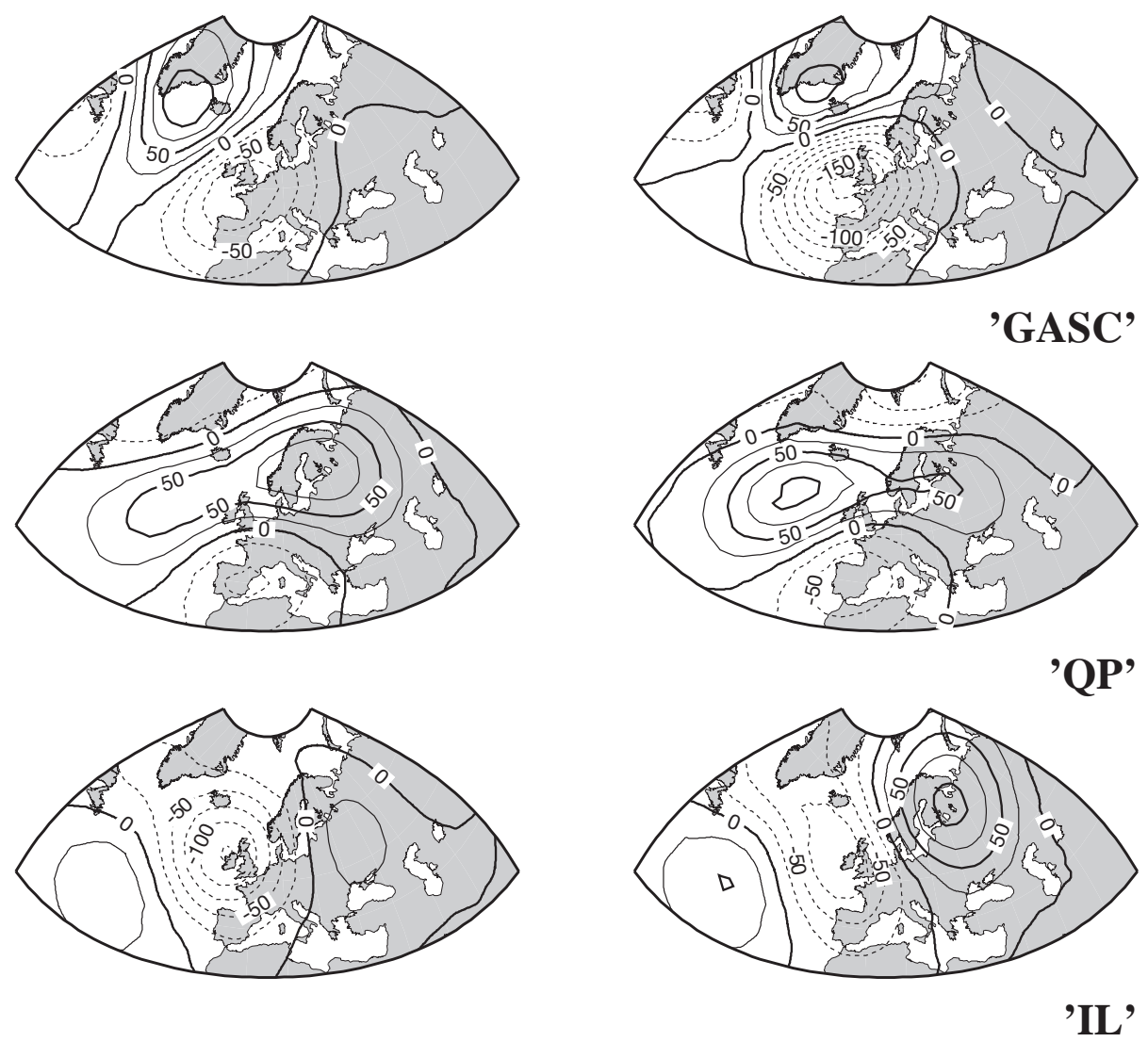

Ticino

Alpes Maritimes

Fig. 7. The 3 cluster centroids found when one classifies $Z 700$ anomaly patterns of all HPEs over Ticino (left-hand anomaly maps) or over the Alpes Maritimes (right-hand maps). GASC: Greenland anticyclone-Sole cyclone; QP: quadrupole; IL: Ireland Low

Maritimes. From a synoptician's point of view, this is not a surprise since both regions are highly exposed to damaging heavy precipitation when southerly flows impinge on the southern flanks of the Alps (Massacand et al. 1998, Ferretti et al. 2000). Still, in fall 2000 it was observed several times that the French Alpes Maritimes events preceded the Ticino events by 12 to $24 \mathrm{~h}$.

Note that almost the same 3 clusters were found using the 17 grid points of the larger domain extending to both sides of the mountain ridge between Ticino and the Rhone valley, although the significance level remained well below $90 \%$. With a Rhone domain made up of the 7 grid points which were discarded because they lie north of the ridge, between the Rhone and Rhine valleys and Ticino, it is interesting to note the emergence of a new cluster with a pronounced low over southern Scandinavia (not shown). This cluster is very similar to the Savoy-Mont Blanc cluster 1 which supplies the most intense snowfalls (winter HPEs) over this domain (Section 3.2).

For brevity, the histograms corresponding to those of Figs $2 \& 3$ are not displayed, but let us say that as for the Alpes Maritimes, cluster 1 (GASC) exhibits the highest discriminating power, since $31 \%$ of the days for which the LSC anomaly pattern correlation with the GASC pattern apcC $>0.8$ are actually HPEs. This percentage drops down to $26 \%$ for $\mathrm{IL}$, and $17 \%$ for QP. There are also many HPEs (more than for the Alpes Maritimes) amongst days with an LSC apcC with the GASC centroid between 0.6 and 0.8 .

\subsection{Roussillon and Riviera Levante}

We also applied the methodology to 2 other Mediterranean border sub-regions. The left-hand column of Fig. 8 shows the 3 clusters responsible for HPEs over the Riviera Levante, that is the part of the Italian Riviera extending roughly from Genoa to Pisa. Once again, they look very similar to the Ticino and Alpes Maritimes clusters of Fig. 7. This is not amazing, since most of the damaging events in the autumn of 2000 indeed affected the 3 sub-regions, but left Roussillon, the French border region north of Catalonia, alone. 

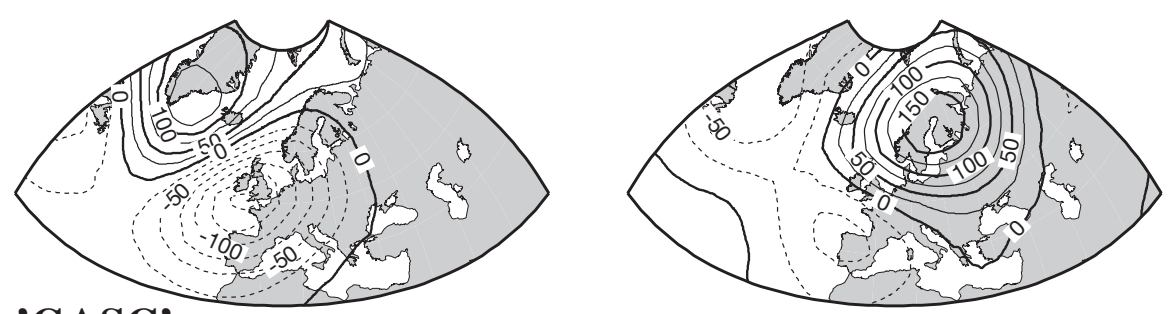

\section{'GASC'}
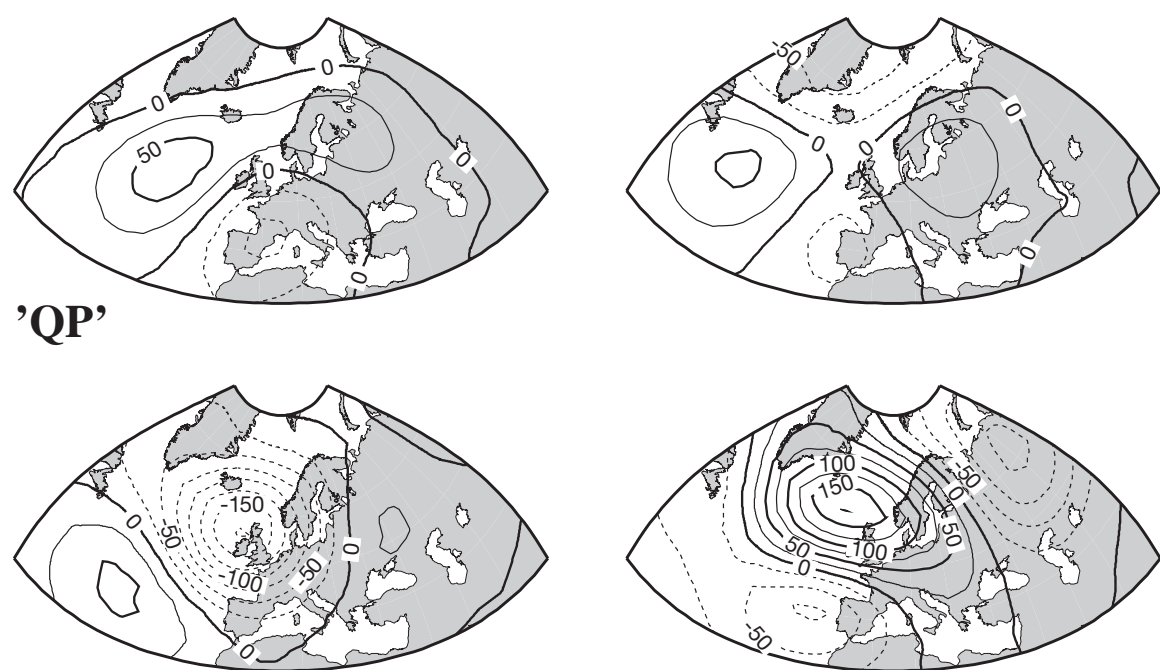

'IL'

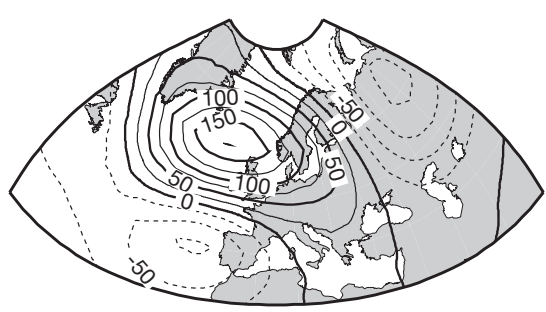

Riviera Levante

Roussillon

Fig. 8. The 3 cluster centroids found when one classifies $Z 700$ anomaly patterns of all HPEs over the Riviera Levante (left-hand column), or Roussillon (right-hand column). GASC, QP and IL only refer to the left-hand column patterns

The HPE LSC cluster centroids for this region (Fig. 8, right column) actually do not correlate with the previous clusters. On the contrary, 2 of them look very similar to blocking-type weather regime patterns (Plaut \& Simonnet 2001). One may be reminded that HPEs multiplied over this region during the negative NAO 19951996 winter. The Roussillon is actually especially exposed to Mediterranean easterlies which often generate intense precipitation over this region and, occasionally, extreme flooding, such as that in midNovember 1999, when precipitation amounts locally exceeded $500 \mathrm{~mm}$ in $24 \mathrm{~h}$.

\subsection{Recent trends of HPEs over Ticino, Roussillon, and Riviera Levante}

Similar to what was observed for the Alpes Maritimes, the Riviera Levante experienced a pronounced negative trend for annual precipitation from HPEs, which decreased by $200 \mathrm{~mm}$ on average between 1971 and 1995 (Fig. 9, left-hand column). The overall de- crease in yearly precipitation, $300 \mathrm{~mm}$, was even more significant, showing that the negative trend affected all types of precipitation, as was the case for the Alpes Maritimes. These features contrast with what happened for Roussillon, where precipitation amounts from HPEs, although limited, were multiplied by 2 during the same period (Fig. 9, right-hand column).

The case of Ticino deserves some more attention. Whereas, with very similar cluster centroids, both the Alpes Maritimes and the Riviera Levante experienced decreasing yearly precipitation amounts, one observes a small positive trend $(+100 \mathrm{~mm})$ for HPE precipitations over Ticino. A plausible explanation is suggested by the consideration of the histograms of Fig. 10, where the fall and winter precipitation (OctoberMarch) have been separated from the summer (AprilSeptember) ones. Precipitation amounts in autumn and winter HPEs over Ticino actually display a light (poorly significant) negative trend, whereas the complementary season (spring-summer) shows a pronounced positive trend $(+200 \mathrm{~mm}$ in $25 \mathrm{yr}$ ), so that one could tentatively solve the apparent contradiction in the following 
Riviera Levante
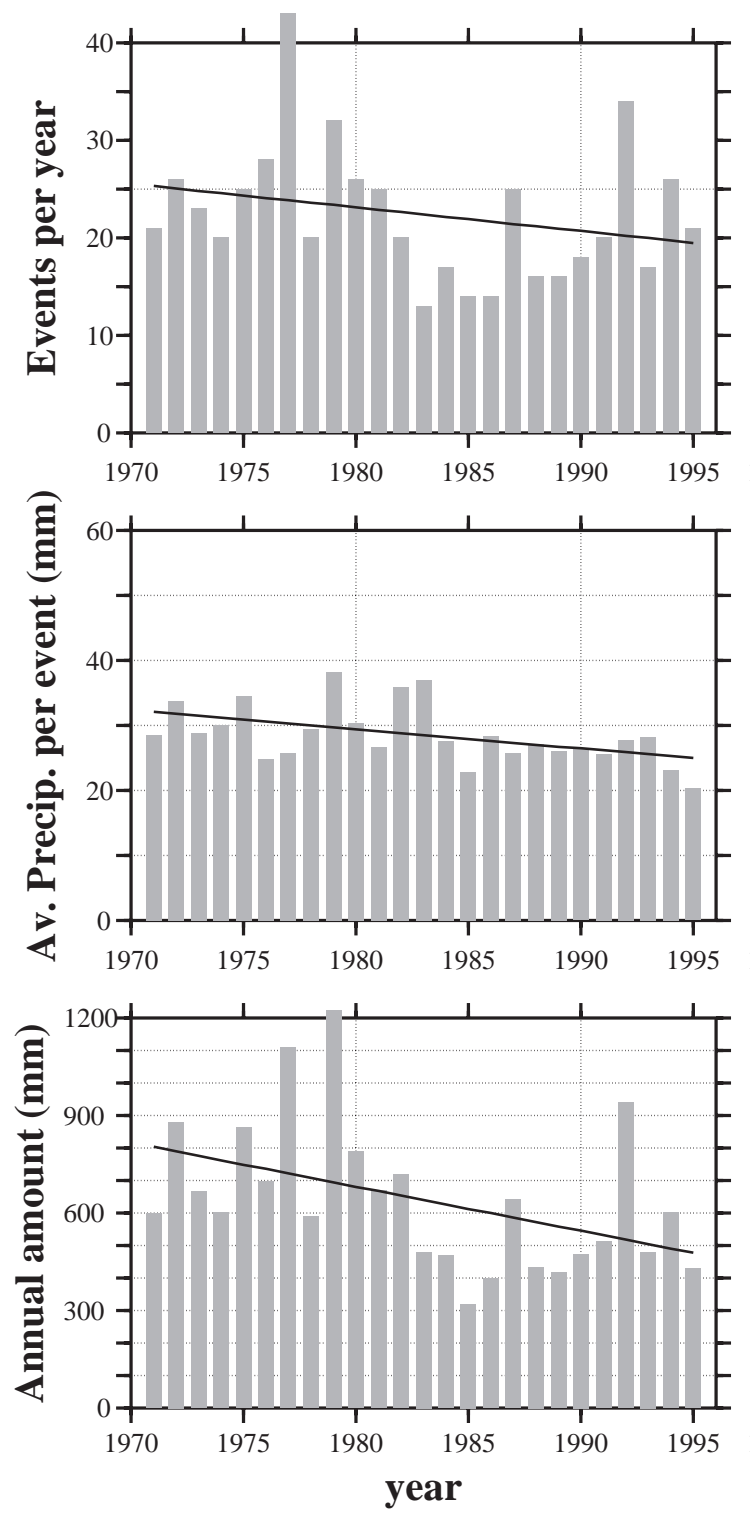

Roussillon
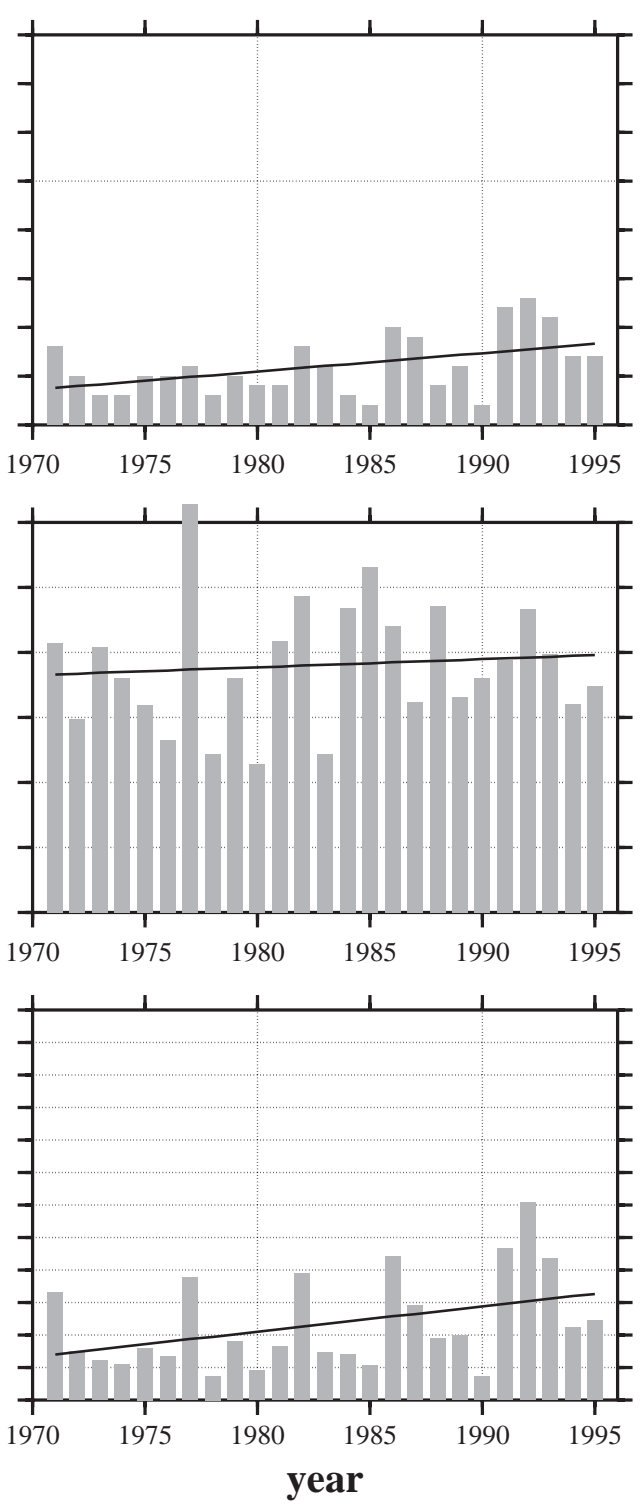

Fig. 9. Number of HPEs per year (top); average precipitation per HPE over the domain (middle), and total annual amount from HPEs, also averaged over the domain gridpoints (bottom), together with the trends. Data from the ETH Zürich Alpine Precipition Climatology

way: during the period 1971-1995, Mediterranean Alpine regions actually tended to experience lower precipitation heights from HPEs: in addition to the summer drought, fall and winter precipitation shortage became a threat. During the cold seasons, rather similar features emerged for Ticino, which, however, received increasing amounts from HPEs during the complementary season when precipitation almost ceases along Mediterranean coastal areas.

As to Roussillon, which also experiences HPEs mostly in the cold season and which is also located along the Mediterranean, the reverse trend is likely to be connected with the very different nature of the large-scale patterns yielding heavy precipitation there. These observations need further investigation in order to isolate the underlying mechanisms.

\section{DISCUSSION}

\subsection{Sensitivity experiments and robustness}

The use of our classifiability index ensures a high significance level for our cluster centroids. With this 
Ticino: Oct.-March
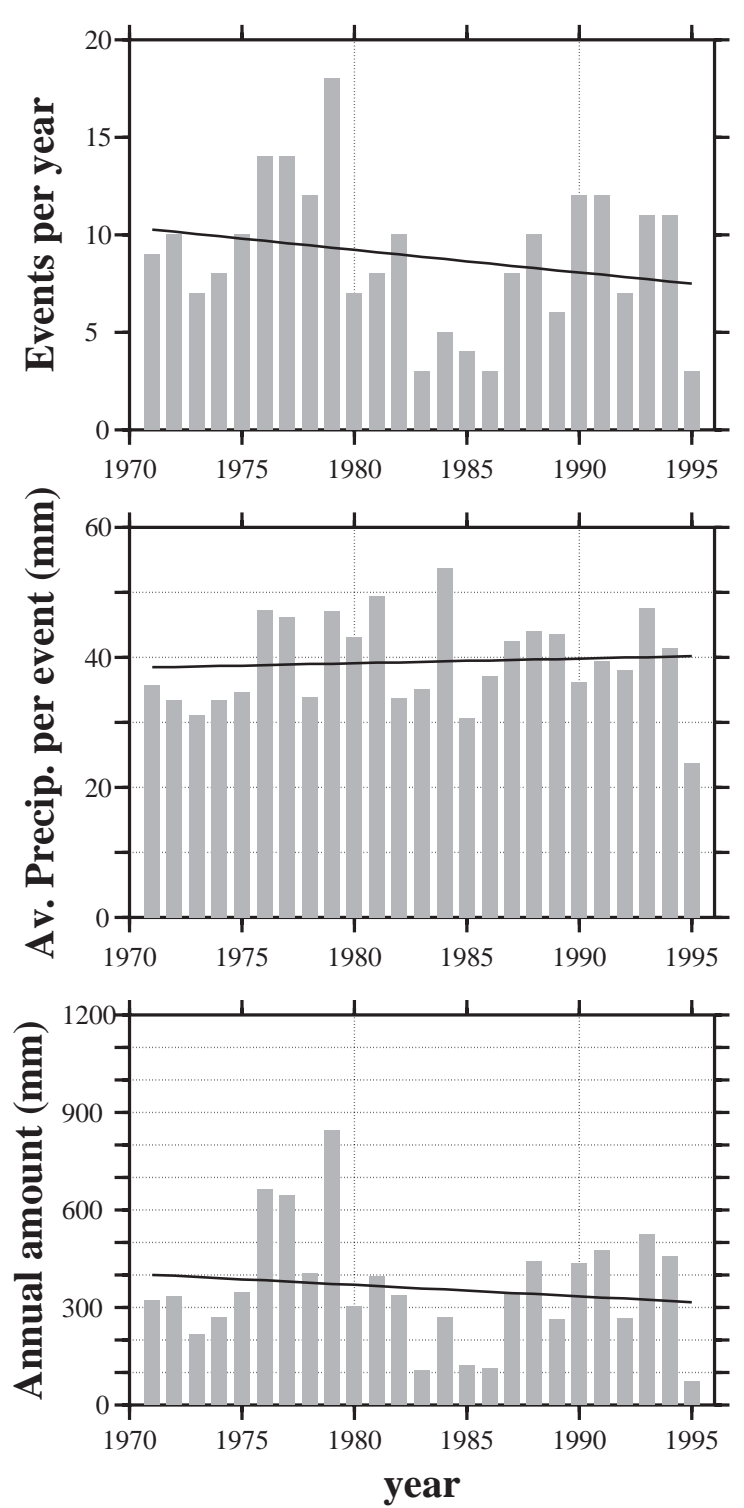

Ticino: April-Sept.
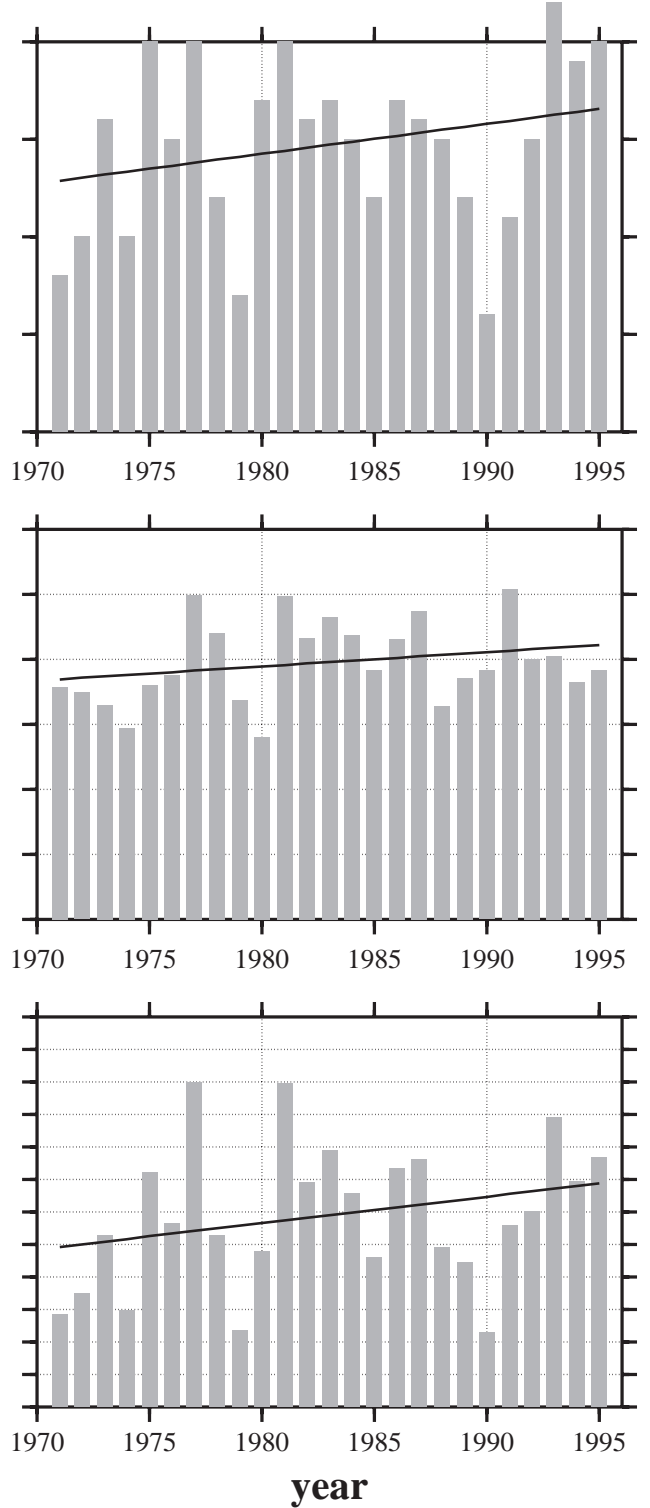

Fig. 10. Number of HPEs per year (top); average precipitation per HPE over the domain (middle), and total annual amount from HPEs, also averaged over the domain gridpoints (bottom), together with the trends. Data from the ETH Zürich Alpine Precipitation Climatology

index higher than the $90 \%$ significance limit, as was the case for all the sub-regions we studied, new classifications starting from new random seeds always led to the same clusters. One may nevertheless ask oneself whether subsets of HPEs would lead to the same patterns. Our choice of a 10-dimensional PC space may also be questioned. In order to test the sensitivity to parameter changes and the robustness of the cluster centroids, we performed 2 categories of sensitivity experiments.

We applied the dynamical cluster algorithm first to HPE LSCs belonging to the first half of the $25 \mathrm{yr}$ period and then, separately, to those belonging to the second half. Finally, the cluster centroids found for the 2 distinct periods were compared. Let us state the main conclusions one can draw in the case of the Alpes Maritimes. For both periods, the same number of clusters, $k=2$, is again selected by the classifiability test. The cluster centroids show only negligible differences between periods 1 and 2. This is particularly obvious for the GASC patterns of Fig. 11a (period 1, first half of the data) and Fig. 11b (second half), which do not differ from the GASC pattern of Fig. 2a corresponding to classification of the full 25 yr HPE LSC set. We show in 
a)
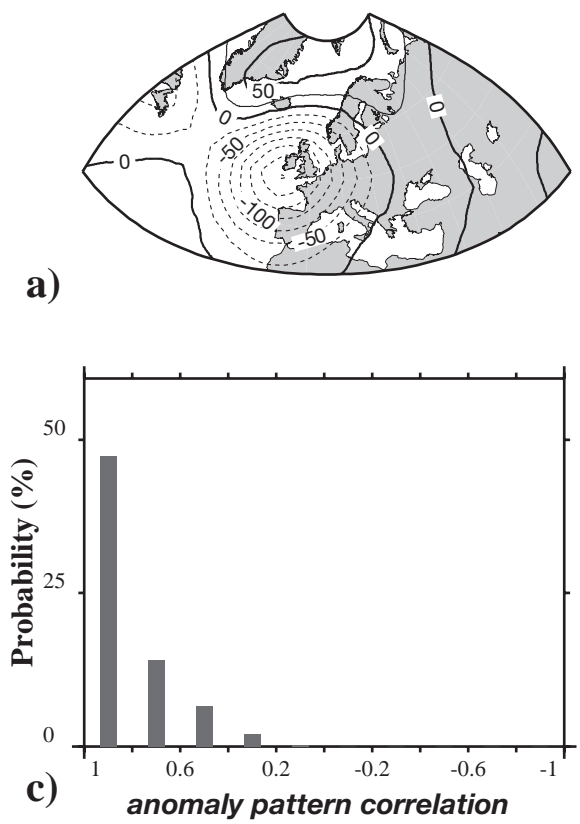

b)
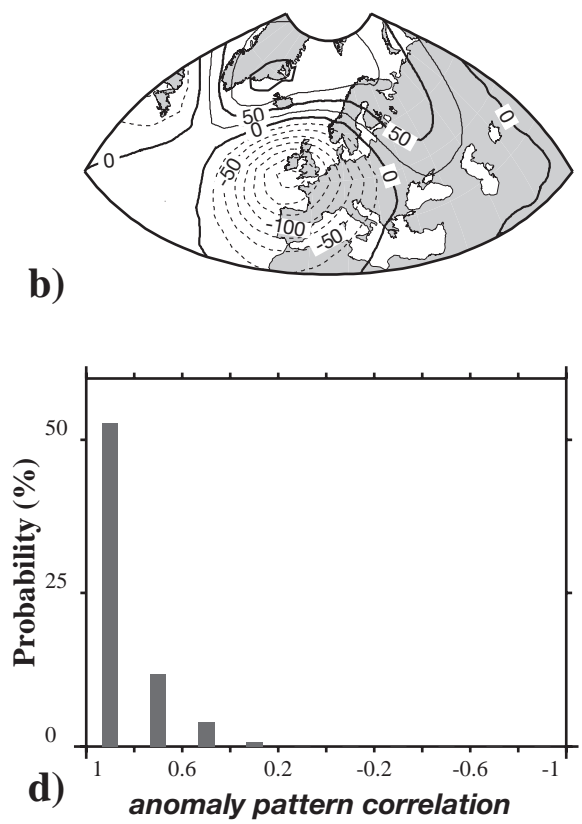

Fig. 11. (a,b) Cluster 1 (GASC) centroid for HPEs over the Alpes Maritimes during (a) the first (1971-June 1983) and (b) the second (July 1983-1995) half-periods. (c,d) Corresponding (conditional) probability of HPE as in Fig. 2, for (c) the first and (d) the second half-periods

Fig. 11c,d the histograms of HPE conditional probability versus the LSC anomaly pattern correlation with GASC (see Section 3.1 for more details). There are only minor differences between the 2 periods, suggesting that the (unknown) uncertainties of the histograms in Fig. 2 are reasonably small. Similar features occur for the rain composites (not shown).

Observations similar to those we made in the particular case above could also be made for the other Alpine sub-regions: there appear only insignificant differences between the cluster centroids for the 2 distinct periods, and indications are given for reasonably small uncertainties of HPE conditional probability histograms as in Fig. 11. In all cases, the clusters with the highest discriminating power (Section 2.4) actually appear fully robust in time.

We also performed sensitivity experiments of another type in order to check the robustness of the classification against the dimension of the PC space in which the classification is performed. Instead of keeping $10 \mathrm{PCs}$, we performed the dynamical cluster algorithm within the 5-dimensional leading PC space. We again state the main conclusions in the case of the Alpes Maritimes; similar conclusions were obtained for the other Alpine sub-regions. With 5 PCs, a number of $k=2$ clusters is again preferred by our statistical test, whereas the patterns (not shown) are obviously the same ones as with 10 PCs. There are, however, minor differences between the sets of cluster centroids with 5 and $10 \mathrm{PCs}$, which may be easily understood: some smaller spatial scale details appearing in Fig. 2 were due to contributions of the PCs of rank higher than 5 and were unavoidably lost when these PCs were discarded.

Hence, our sensitivity experiments firmly establish the robustness of the HPE LSC cluster centroids. For instance, the same number of clusters and the same patterns are found using the first or second half-period (12.5 yr) HPEs alone. The exact number of PCs kept to perform the classification is also irrelevant.

\subsection{Weather regimes, the NAO, and HPEs}

We observed in previous sections that the classification of HPE-only LSCs often leads to clusters with a high discriminating power. But one may ask whether there also exist significant connections between the socalled weather regimes, the most recurrent LSC patterns (Plaut \& Simonnet 2001, and references therein) and local HPEs. For the sake of brevity, we again limit the discussion to the Alpes Maritimes; similar conclusions may be drawn for other sub-Alpine domains. The histograms in Fig. 12 display the conditional probability of HPE occurrence versus the anomaly pattern correlation between daily LSCs and the weather regime centroids of (Plaut \& Simonnet 2001). They have to be compared with those of Fig. 2. With the weather regimes, the conditional probability never significantly increases 
above its climatological mean indicated by the thin horizontal line, and it is obvious that none of the weather regime patterns is a suitable one if one intends to forecast HPEs through a downscaling algorithm. By contrast (Section 3.1), the HPE conditional probability may be enhanced by a factor of 15 for days whose LSCs correlate with GASC, with an apcc > 0.8.

Let us add a few words about a possible correlation between HPEs and the NAO index. We computed correlations between HPE precipitation and this index globally, as well as for winter, or even per calendar month. All the figures were found to be surprisingly small, even for the SavoyMont Blanc region (-0.06 over the whole year, +0.07 for winter). The explanation is likely to lie in the fact that the value of the NAO index results from some mean monthly or seasonal behaviour, whereas HPEs are indeed rare events. The HPE LSCs yield almost negligible contributions to seasonal means, which receive their most outstanding contributions from the most frequently occurring patterns, the weather regimes, such as $\mathrm{ZO}$, the prevailing LSC pattern during periods with a large positive NAO index. Moreover, one could verify that the HPE cluster centroids are almost uncorrelated with the weather regime patterns. For instance, notwithstanding some common belief, the cluster 1 centroid of Fig. 4a (the cluster responsible for most of February 1999's damaging events over the northern Alps) is only very weakly correlated with ZO. In the case of the Alpes Maritimes, the weak negative correlation between HPE precipitations and the NAO index $(-0.22$ during fall, -0.17 for winter), is also hardly significant.

\subsection{Other approaches}

We finally briefly discuss a few alternative ways of dealing with HPE LSCs.

- Compositing all HPE LSCs. With such an approach, smaller LSC domains are preferred since, in most cases, significant composite anomalies only extend over a limited geographical area. Fig. 13 displays all HPE composite maps for both the Savoy and the Alpes Maritimes. At first glance, the composite approach seems rather interesting in the latter
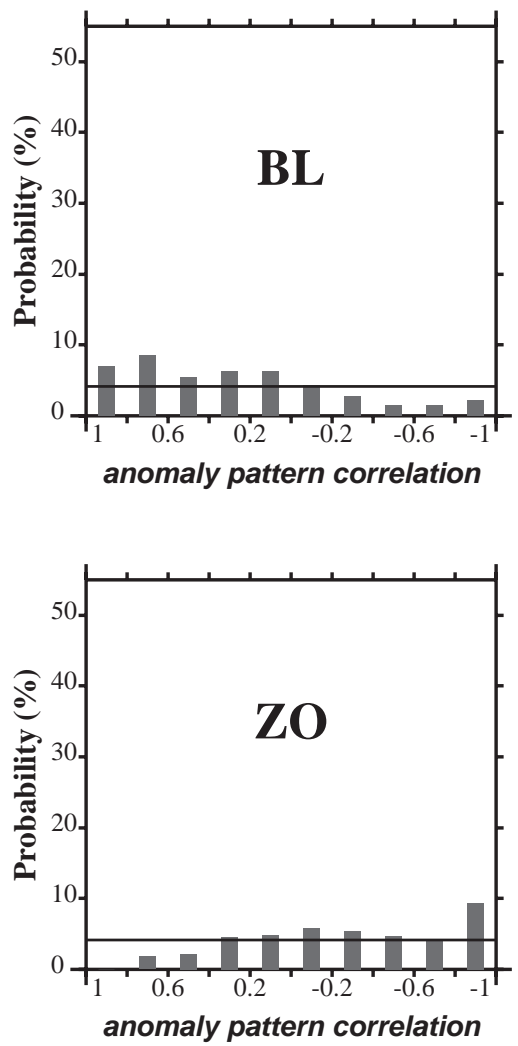

anomaly pattern correlation

Fig. 12. HPE (conditional) probability over the Alpes Maritimes against the anomaly pattern correlation of daily LSCs with the 4 Z700 weather regime centroids of Plaut \& Simonnet (2001). AR: Atlantic Ridge; BL: Blocking; GA: Greenland Anticyclone; ZO: zonal flow

case: any day with an apcc $>0.8$ with the composite pattern of Fig. 13d has a high probability of being an HPE, and the grid point precipitation histogram of Fig. 13f exhibits a sharp peak for the highest apcc values. After closer inspection (not shown), one realizes however that the number of days with an LSC apcc $>0.8$ with the composite pattern of Fig. 13d is so small that this apparently higher discriminating power is almost irrelevant as compared to that of the GASC cluster. Other advantages plead in favour of the cluster approach: (1) the different seasonal behaviour shown by different clusters (Fig. 3); (2) the more subtle understanding of the underlying dynamical processes which is made possible through the classification approach: remember in particular the insight one gets into the origin of the opposite trends exhibited by the Queyras clusters 1 and 2.

- Taking a narrower domain never improved the cluster properties; they were at best almost unchanged; some Alpine sub-regions were more sensitive to the western parts of the domain, other ones to the eastern part. 
a)
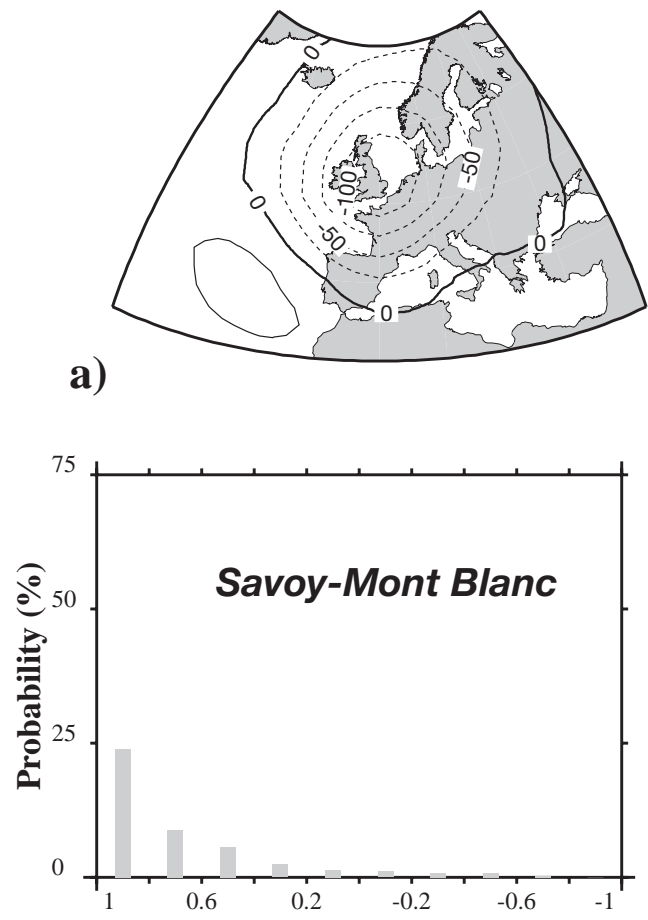

b) anomaly pattern correlation

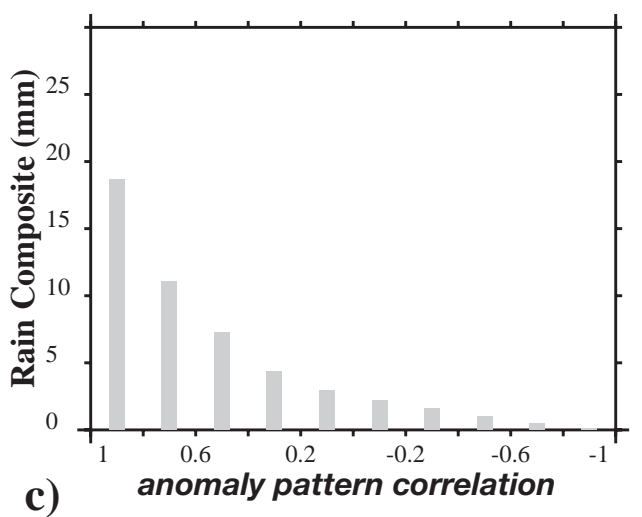

d)
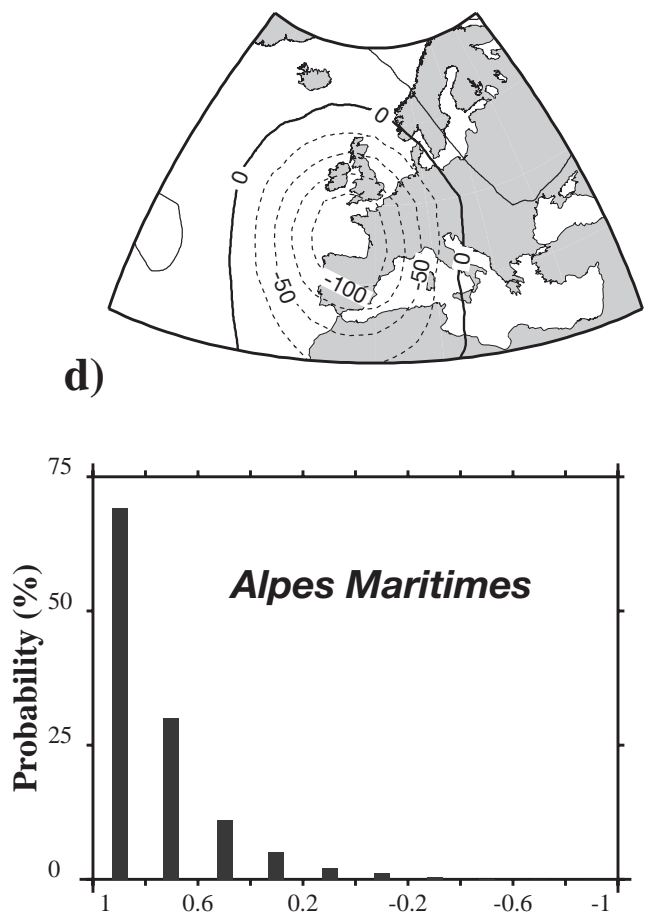

e) anomaly pattern correlation

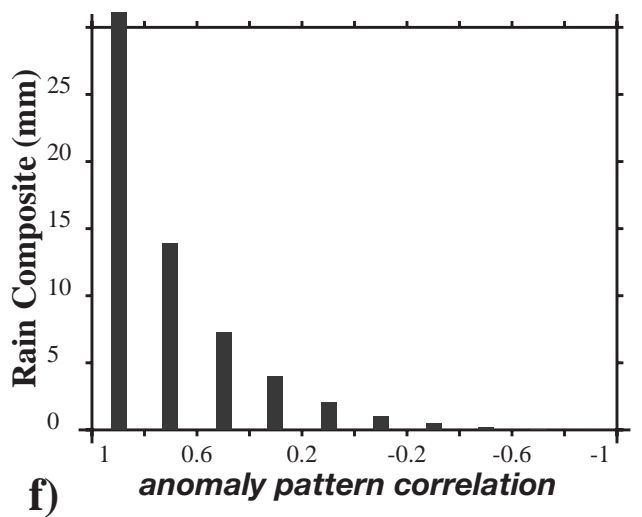

Fig. 13. $(a, d)$ Composite geopotential anomaly for all HPEs; (b,e) HPE conditional probability vs anomaly pattern correlation between any day's LSC and the previous composite; $(\mathrm{c}, \mathrm{f})$ all days of the $25 \mathrm{yr}$ precipitation composite for the (a-c) Savoy-Mont Blanc and $(\mathrm{d}-\mathrm{f})$ Alpes Maritimes domain grid points marked with a larger circle in Fig. 1

- Taking SLP as the LSC field, instead of the Z700 heights, tends to make cluster discriminating power a little lower.

- Other possibilities, such as the choice of thickness fields, never brought improvements of the cluster discriminating power.

\section{SUMMARY AND CONCLUSIONS}

If one's motivation lies in a need for a description of local climate better than mere average climate, or for downscaling algorithms appropriate for local sensitive empirical weather forecasts in most common situations, the classification of all daily LSCs into a small number of classes, the so-called weather regimes, provides a very attractive approach (Plaut \& Simonnet 2001). However, if one's main interest lies in somewhat rare events, such as HPEs, an approach where one only classifies LSCs for HPE days proves much more relevant. Of course the cluster centroids one obtains are situated in less populated regions of the space spanned by the leading PCs (there are, for instance, about 6 times less daily LSCs with an apcC $>0.8$ with the GASC cluster centroid than with the ZO weather regime), but their interest relies in the corresponding 
highly enhanced probability of HPEs. Anomaly pattern correlations are almost negligible between these LSC cluster centroids and the weather regime patterns, which provides a plausible explanation for the also negligible observed correlation between climatic indices such as the NAO and heavy precipitation over most Alpine sub-regions. These clusters of subregional scale HPE LSCs are fully 'data adaptive'. The cluster centroids are objectively established, which contrasts with numerous downscaling approaches where one classifies according to some a priori established criterion, such as the flow orientation etc.

In order to check the robustness of the classifications, we introduced a 'classifiability index'. The cluster centroids were found to be very robust for all the studied Alpine sub-regions. In all cases, our index allowed us to unambiguously fix the number of clusters. Additional sensitivity experiments were nevertheless performed. They firmly established the robustness of the cluster centroids against reasonable changes in the procedure: the same patterns are indeed recovered using separate data from the first or the second halfperiod. The exact number of PCs kept to perform the classification is also irrelevant.

While very different patterns correspond to the Alpes Maritimes, the Savoy-Mont Blanc, or the Roussillon for instance, the cluster centroids for Ticino or the Riviera Levante could be put in a one-to-one correspondence with those for the Alpes Maritimes, which is not very amazing since the same events often affect these 3 regions within 1 or $2 \mathrm{~d}$. We tentatively defined a linear trend for each sub-region, putting together all the precipitation due to HPEs. For the period 1971-1995 covered by the Alpine Precipitation Climatology dense data set, opposite trends appeared over regions on the northern and southern flanks of the Alps: annual precipitation due to HPEs began with roughly 2.5 times more over the Alpes Maritimes when compared to the Savoy-Mont Blanc, whereas they end with similar values for both regions in the investigated period. The total precipitation amounts display even more pronounced opposite trends. Correlations with the NAO index could hardly be invoked since they were found to be negligibly small. Over Ticino, there has been an increase in spring and summer precipitation, a period of the year during which HPEs become rare in the Mediterranean coastal areas. A positive trend was also observed over Roussillon, where HPEs are mainly due to Mediterranean easterlies.

Given the high discriminating power of some cluster centroids, such as GASC, and the weakness of direct deterministic HPE forecasts in many cases, downscaling algorithms using amongst others the forecasted LSCs and the HPE LSC clusters could provide very useful alternatives. In any case, they could at least allow one to examine the possible meso-scale consequences of global change as simulated by GCMs in a statistical way.

Acknowledgements. We especially thank C. Frei and C. Schär, ETH Zürich, for the Alpine Precipitation Climatology. We also thank the CRU for making the LSC fields they extracted from the NCEP reanalyses available to us.

\section{LITERATURE CITED}

Bardossy A, Plate EJ (1992) Space-time model for daily rainfall using atmospheric circulation patterns. Water Resour Res 28:1247-1259

Buzzi A, Tartaglione N, Malguzzi P (1998) Numerical simulations of the 1994 Piedmont flood. Role of orography and moist processes. Mon Weather Rev 126:2369-2383

Doswell CA, Ramis C, Romero R, Alonso S (1998) A diagnostic study of three heavy precipitation episodes in the western Mediterranean region. Weather Forecast 13: 102-124

Emmanuel KA (1991) A scheme for representing cumulus convection in large scale models. J Atmos Sci 21: 2313-2335

Ferretti R, Low-Nam S, Rotunno R (2000) Numerical simulations of the Piedmont flood of 4-6 November 1994. Tellus 52A:162-180

Frei C, Schär C (1998) A precipitation climatology for the Alps from high-resolution rain-gauge observations. Int J Climatol 18:873-900

Hay LE, McCabe GJ, Wolock DM, Ayers MA (1991) Simulation of precipitation by weather type analysis. Water Resour Res 27:493-501

Hess P, Brezowsky H (1952) Katalog der Grosswetterlagen Europa. Ber Dtsch Wetterdienst 3, Bad Kissingen

Hess P, Brezowsky H (1969) Katalog der Grosswetterlagen Europa. Ber Dtsch Wetterdienst 113, Offenbach am Main

Jones PD (1994) Hemispheric surface air temperature variations: a reanalysis and an update to 1993. J Clim 7: 1794-1802

Kalnay E, Kanamitsu M, Kistler R, Collins W (1996) The NCEP/NCAR 40-year Reanalysis Project. Bull Am Meteorol Soc 77:437-471

Katz RW, Parlange MB (1996) Mixture of stochastic processes: application to statistical downscaling. Clim Res 7:185-193

Lamb HH (1972) British Isles weather types and a register of a daily sequence of circulation patterns, 1861-1971. Geophys Mem 116

Massacand AC, Wernli H, Davies HC (1998) Heavy precipitation on the Alpine southside: an upper-level precursor. Geophys Res Lett 25:1435-1438

Michelangeli PA, Vautard R, Legras B (1995) Weather regimes: recurrence and quasi stationarity. J Atmos Sci 52: $1237-1256$

Plaut G, Simonnet E (2001) Large-scale circulation classification, weather regimes, and local climate over France, the Alps, and Western Europe. In: von Storch H, Jones P (eds) ACCORD: Atmospheric circulation classification and regional downscaling. CR SPECIAL 9. Clim Res 17:303-324

Romero R, Ramis C, Alonso S, Doswell CA, Stensud DJ (1998) Mesoscale model simulations of three heavy precipitation events in the western Mediterranean region. Mon Weather Rev 126:1859-1881

Widmann ML (1996) Mesoscale variability and long term 
trends of Alpine precipitaion and their relation to synoptic-scale flow. Dissertation ETH No. 11769, Zürich

Wilby RL (1998) Downscaling of daily precipitation using daily airflow and seasonal teleconnection indices. Clim Res 10:163-178

Wilby RL, Wigley TML, Conway D, Jones PD, Hewiton BC,
Main J, Wilks DS (1998) Statistical downscaling of general circulation model output: a comparison of methods. Water Resour Res 34:2995-3008

Wilks DS, Wilby RL (1999) The weather generation game: a review of stochastic weather models. Prog Phys Geogr 23: $329-357$

Proofs received from author(s): August 27, 2001 